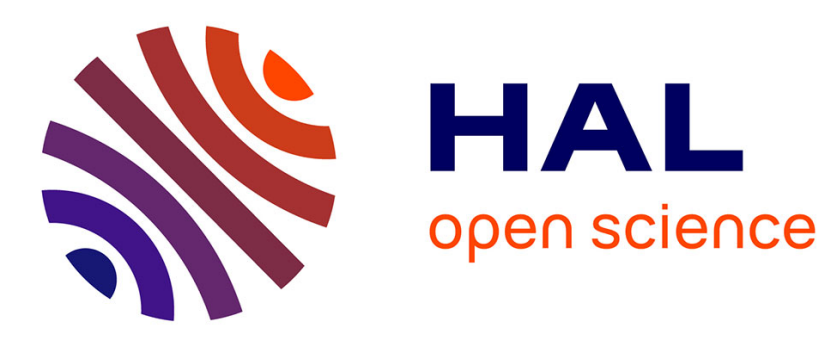

\title{
Fuzzy Measures and Integrals: Recent Developments Michel Grabisch
}

\section{To cite this version:}

Michel Grabisch. Fuzzy Measures and Integrals: Recent Developments. D. E. Tamir and D. Rishe and A. Kande. Fifty Years of Fuzzy Logic and its Applications, 2015, 10.1007/978-3-319-19683-1_8 . hal-01302377

\section{HAL Id: hal-01302377 \\ https://hal.science/hal-01302377}

Submitted on 14 Apr 2016

HAL is a multi-disciplinary open access archive for the deposit and dissemination of scientific research documents, whether they are published or not. The documents may come from teaching and research institutions in France or abroad, or from public or private research centers.
L'archive ouverte pluridisciplinaire HAL, est destinée au dépôt et à la diffusion de documents scientifiques de niveau recherche, publiés ou non, émanant des établissements d'enseignement et de recherche français ou étrangers, des laboratoires publics ou privés. 


\title{
Fuzzy measures and integrals: recent developments
}

\author{
Michel GRABISCH \\ Paris School of Economics, University of Paris I, 106-112, Bd. de l'Hôpital, 75013 Paris, France. \\ Tel. (+33)14407-8285 michel.grabisch@univ-paris1.fr
}

\begin{abstract}
This paper surveys the basic notions and most important results around fuzzy measures and integrals, as proposed independently by Choquet and Sugeno, as well as recent developments. The latter includes bases and transforms on set functions, fuzzy measures on set systems, the notion of horizontal additivity, basic Choquet calculus on the nonnegative real line introduced by Sugeno, the extension of the Choquet integral for nonmeasurable functions, and the notion of universal integral.
\end{abstract}

\section{Introduction}

This paper gives a survey of the research done on fuzzy measures and integrals since Sugeno proposed in 1974 the concept of fuzzy measure, with an emphasis on recent results. This field of research lies at the intersection of several independent domains, which makes it very active and attractive, namely, measure theory, theory of aggregation functions, cooperative game theory, combinatorial optimization, pseudo-Boolean functions and more generally theoretical computer sciences. As an illustration of this fact, the word "fuzzy measure" which was coined by Sugeno, has many different names according to the field where it is used: nonadditive measure, capacity, monotone game, pseudo-Boolean function, rank function of a polymatroid, etc. Evidently, this short paper cannot make a complete account of all the research undertaken in this area, a whole book will hardly suffices. Indeed, the author is preparing a monograph on this topic, with the title: "Set functions, games and capacities in decision making", to be published by Springer around the end of 2015. This paper gives a kind of quick and necessarily simplified summary of selected topics. We recommend the interested reader to consult the main (available) monographs dealing with fuzzy measures and integrals: Pap [1], Denneberg [2], Wang and Klir [3], the Handbook of measure theory edited by Pap [4], as well as the edited book [5], and the survey paper [6]. The latter focusses on application in multicriteria decision making, an aspect which is not covered by this paper, restricting to theory.

To avoid intricacies, in the whole paper the universal set $X$ is finite, with $|X|=n$. We often use $\vee, \wedge$, which collapse to maximum and minimum on finite sets.

\section{Fuzzy measures}

Fuzzy measures introduced by Sugeno [7] are generalization of classical measures, i.e., additive and nonnegative set functions, whose domain is an algebra $\mathcal{F}$ on $X$. As we will see in Section 2.4, the structure of algebra is not needed here, and various structures can be thought of. For simplicity, we assume $\mathcal{F}=2^{X}$ in the first subsections, the general case will be addressed in the last one. 


\subsection{Definition, main families and properties}

A fuzzy measure on $X$ is a set function $\mu: 2^{X} \rightarrow \mathbb{R}$ such that $\mu(\varnothing)=0$ and $\mu$ obeys monotonicity: $A \subseteq B \subseteq X$ implies $\mu(A) \leqslant \mu(B)$. Fuzzy measures are also called capacities (after Choquet [8]), nonadditive measures (Denneberg [2]), monotone measures (Wang and Klir [3]), etc. If in addition $\mu(X)=1$, then the fuzzy measure is said to be normalized.

If monotonicity is dropped from the definition, we obtain nonmonotonic fuzzy measures, more commonly called games, denoted usually by $v$.

One of the most important property of fuzzy measures (or games as well) is convexity, a.k.a. supermodularity. A fuzzy measure $\mu$ is convex if for all $A, B \in 2^{X}, \mu(A \cup B)+\mu(A \cap$ $B) \geqslant \mu(A)+\mu(B)$. If the reverse inequality holds, $\mu$ is said to be concave or submodular. Convexity is generalized by the so-called $k$-monotonicity property: $\mu$ is $k$-monotone for some fixed $2 \leqslant k \leqslant n$ if for any family of $k$ sets $A_{1}, \ldots, A_{k} \in 2^{X}$,

$$
\mu\left(\bigcup_{i=1}^{k} A_{i}\right) \geqslant \sum_{\substack{I \subseteq\{1, \ldots, k\} \\ I \neq \varnothing}}(-1)^{|I|+1} \mu\left(\bigcap_{i \in I} A_{i}\right) .
$$

Moreover, $\mu$ is totally monotone if it is $k$-monotone for every $k \geqslant 2$ (in fact, $2 \leqslant k \leqslant 2^{n}-2$ suffices). The k-alternating property is defined similarly, interchanging $\bigcap$ and $\bigcup$ and reversing inequality. Lastly, $\mu$ is said to be maxitive if $\mu(A \cup B)=\mu(A) \vee \mu(B)$, and minitive if $\mu(A \cap B)=\mu(A) \wedge \mu(B)$.

The simplest fuzzy measures which can be thought of are 0-1-fuzzy measures: their range is simply $\{0,1\}$. In game theory, they are called simple games and are useful in voting theory. Among them, particularly useful are unanimity games (a.k.a. simple support functions): for any $\varnothing \neq A \subseteq X$, the unanimity game $u_{A}$ is defined by

$$
u_{A}(B)= \begin{cases}1, & \text { if } B \supseteq A \\ 0, & \text { otherwise. }\end{cases}
$$

The next remarkable families are possibility and necessity measures: a possibility (resp., necessity) measure is a normalized maxitive (resp., minitive) fuzzy measure (Zadeh, [9], Dubois and Prade [10]). Necessity measures are particular cases of belief functions, as proposed by Shafer [11] (similarly, plausibility functions generalize possibility measures). Mathematically speaking, a belief (resp., plausibility) function is a normalized totally monotone (resp., alternating) fuzzy measure.

\subsection{Transforms and bases}

The set of games, as well as the set of set functions, form a vector space of dimension $2^{n}-1$ (resp., $2^{n}$ ). This is not the case for the set of fuzzy measures, which is only a cone, while the set of normalized capacities is a polytope, whose vertices are the $0-1$ fuzzy measures (Stanley [12], Radojevic [13]). In the rest if this section, we deal with the vector space of set functions (the results can be however easily adapted to the set of games).

A transform is a mapping $\Psi: \mathbb{R}^{\left(2^{N}\right)} \rightarrow \mathbb{R}^{\left(2^{N}\right)}$, assigning to any set function $\xi$ the set function $\Psi^{\xi}$. If the transform is linear and invertible, then it induces a basis of the vector space of set functions (and similarly for games). Conversely, any basis induces a linear invertible transformation. This is explicited in the next lemma. 
Lemma 1. (Faigle and Grabisch [14]) For every basis $\left\{b_{S}\right\}_{S \in 2^{X}}$ of $\mathbb{R}^{2^{X}}$, there exists a unique linear invertible transform $\Psi$ such that for any $\xi \in \mathbb{R}^{2^{X}}$,

$$
\xi=\sum_{S \in 2^{X}} \Psi^{\xi}(S) b_{S}
$$

whose inverse $\Psi^{-1}$ is given by $\xi \mapsto\left(\Psi^{-1}\right)^{\xi}=\sum_{T \in 2^{X}} \xi(T) b_{T}$.

Conversely, to any transform $\Psi$ corresponds a unique basis $\left\{b_{S}\right\}_{S \in 2^{X}}$ such that (2) holds, given by $b_{S}=\left(\Psi^{-1}\right)^{\delta_{S}}$, where $\delta_{S}$ is a 0-1-valued set function defined by $\delta_{S}(T)=1$ if and only $T=S$.

It is well known that the set of unanimity games forms a basis of the set of games. Adding the 0 -1-valued set function $u_{\varnothing}$ defined by $u_{\varnothing}(S)=1$ if and only if $S=\varnothing$, we get a basis for the vector space of set functions. By Lemma 1, the corresponding transform, denoted by $m$, satisfies

$$
\xi(A)=\sum_{B \subseteq A} m^{\xi}(B) \quad\left(A \in 2^{X}\right)
$$

which yields

$$
m^{\xi}(A)=\sum_{B \subseteq A}(-1)^{|A \backslash B|} \quad\left(A \in 2^{X}\right) .
$$

This transform is known as the Möbius transform, famous in combinatorics. Among the many existing transforms, at least two of them have a special interest. The interaction transform [15], generalizing the Shapley value [16] and the interaction index of Murofushi and Soneda [17], has the following expression:

$$
I^{\xi}(A):=\sum_{B \subseteq X \backslash A} \frac{(n-b-a) ! b !}{(n-a+1) !} \Delta_{A} \xi(B)=\sum_{K \subseteq X} \frac{|X \backslash(A \cup K)| !|K \backslash A| !}{(n-a+1) !}(-1)^{|A \backslash K|} \xi(K)
$$

for all $A \subseteq X$, where $a, b, k$ are cardinalities of subsets $A, B, K$, respectively, and $\Delta_{A} \xi(B)=$ $\sum_{K \subseteq A}(-1)^{|A \backslash K|} \xi(B \cup K)$. This transform enables the interpretation of fuzzy measures in a multicriteria decision making context $[18,6]$. The inverse transform is given by

$$
\left(I^{-1}\right)^{\xi}(S)=\sum_{K \subseteq X} \beta_{|S \cap K|}^{|K|} \xi(K)
$$

with coefficients $\beta_{k}^{l}$ given by

$$
\beta_{k}^{l}=\sum_{j=0}^{k}\left(\begin{array}{l}
k \\
j
\end{array}\right) B_{l-j} \quad(k \leqslant l),
$$

where the $B_{j}$ 's are the Bernoulli numbers. It follows from Lemma 1 that the corresponding basis is

$$
b_{T}^{I}(S)=\beta_{|T \cap S|}^{|T|} \quad\left(S, T \in 2^{X}\right) .
$$

The interaction transform of $\xi$ can be expressed in a simple way through its Möbius transform:

$$
I^{\xi}(A)=\sum_{B \supseteq A} \frac{1}{b-a+1} m^{\xi}(B) .
$$


The second transform of interest is the so-called Fourier transform, well known in computer sciences (see, e.g., de Wolf [19] and O'Donnell [20]). The Fourier transform of a set function $\xi$ is defined by

$$
F^{\xi}(S)=\frac{1}{2^{n}} \sum_{K \subseteq X}(-1)^{|S \cap K|} \xi(K) .
$$

Interestingly enough, it is auto-inverse up to the factor $1 / 2^{n}$ :

$$
\left(F^{-1}\right)^{\xi}(S)=\sum_{K \subseteq X}(-1)^{|S \cap K|} \xi(K)
$$

The corresponding basis is therefore

$$
b_{T}^{F}(S)=\sum_{K \subseteq X}(-1)^{|S \cap K|} \delta_{T}(K)=(-1)^{|S \cap T|} \quad\left(S, T \in 2^{X}\right) .
$$

The vectors of this basis (not that these are not games) are called parity functions in the literature of computer sciences. They are up to a recoding equal to the Walsh functions $w_{S}(T)=(-1)^{|S \backslash T|}$ (indeed, $b_{T}^{F}(S)=w_{S}(X \backslash T)$ ). These are a finite version of the original functions proposed by Walsh (see Hurst et al. [21]), who form a orhonormal basis of the set of square integrable functions on $[0,1]$. The major advantage of the Fourier (or Walsh) basis is that it is orthonormal, in the sense that $\left\langle b_{T}^{F}, b_{S}^{F}\right\rangle=1$ if $S=T$, and 0 otherwise, where the inner product is defined by

$$
\left\langle\xi, \xi^{\prime}\right\rangle=\frac{1}{2^{n}} \sum_{S \in 2^{X}} \xi(S) \xi^{\prime}(S) .
$$

Another remarkable property is that the Fourier transform turns the convolution product into an ordinary product (like with the original definition of the Fourier transform):

$$
F^{\xi * \xi^{\prime}}=F^{\xi} F^{\xi^{\prime}}
$$

where the convolution product of two set functions is defined by

$$
\left(\xi * \xi^{\prime}\right)(S)=\frac{1}{2^{n}} \sum_{T \in 2^{X}} \xi(S \Delta T) \xi^{\prime}(T)
$$

( $S \Delta T$ is the symmetric difference, i.e., $(S \cup T) \backslash(S \cap T)$ ).

We finish this section by giving the bounds of the Möbius transform for a normalized fuzzy measure. Surprisingly, the interval in which the Möbius transform of a normalized fuzzy measure can vary is not $[-1,1]$, but its bounds grow rapidly with $n$, approximately in $\frac{4^{\frac{n}{2}}}{\sqrt{\frac{\pi n}{2}}}$, as shown in [22] (corrected version of an earlier publication [23]). The precise result is as follows.

Theorem 1. For any normalized fuzzy measure $\mu$, its Möbius transform satisfies for any $A \subseteq N,|A|>1$ :

$$
-\left(\begin{array}{c}
|A|-1 \\
l_{|A|}^{\prime}
\end{array}\right) \leqslant m^{\mu}(A) \leqslant\left(\begin{array}{c}
|A|-1 \\
l_{|A|}
\end{array}\right),
$$


with

$$
l_{|A|}=2\left\lfloor\frac{|A|}{4}\right\rfloor, \quad l_{|A|}^{\prime}=2\left\lfloor\frac{|A|-1}{4}\right\rfloor+1
$$

and for $|A|=1<n$ :

$$
0 \leqslant m^{\mu}(A) \leqslant 1
$$

and $m^{\mu}(A)=1$ if $|A|=n=1$. These upper and lower bounds are attained by the normalized fuzzy measures $\mu_{A}^{*}, \mu_{A *}$, respectively:

$\mu_{A}^{*}(B)=\left\{\begin{array}{ll}1, & \text { if }|A|-l_{|A|} \leqslant|B \cap A| \leqslant|A| \\ 0, & \text { otherwise }\end{array}, \quad \mu_{A *}(B)= \begin{cases}1, & \text { if }|A|-l_{|A|}^{\prime} \leqslant|B \cap A| \leqslant|A| \\ 0, & \text { otherwise }\end{cases}\right.$ for any $B \subseteq N$.

We give in Table 1 the first values of the bounds.

\begin{tabular}{|l|rrrrrrrrrrrr|}
\hline$|A|$ & 1 & 2 & 3 & 4 & 5 & 6 & 7 & 8 & 9 & 10 & 11 & 12 \\
\hline u.b. of $m^{\mu}(A)$ & 1 & 1 & 1 & 3 & 6 & 10 & 15 & 35 & 70 & 126 & 210 & 462 \\
\hline l.b. of $m^{\mu}(A)$ & $1(0)$ & -1 & -2 & -3 & -4 & -10 & -20 & -35 & -56 & -126 & -252 & -462 \\
\hline
\end{tabular}

Table 1. Lower and upper bounds for the Möbius transform of a normalized fuzzy measure

\section{$2.3 \quad k$-additive and $p$-symmetric fuzzy measures}

A fuzzy measure $\mu$ is additive if $\mu(A \cup B)=\mu(A)+\mu(B)$ for every disjoint $A, B \in$ $2^{X}$. Normalized additive fuzzy measures therefore coincide with probability measures. Observing that the Möbius transform of an additive fuzzy measure $\mu$ satisfies $m^{\mu}(A)=0$ for all $A \in 2^{X}$ such that $|A|>1$, a natural generalization of additivity is $k$-additivity: a fuzzy measure $\mu$ is $k$-additive $(1 \leq k \leq n)$ if $m^{\mu}(A)=0$ for all $A \in 2^{X}$ such that $|A|>k$, and there exists at least one $A \in 2^{X}$ such that $m^{\mu}(A) \neq 0$ (Grabisch [15]). It follows that a $k$-additive fuzzy measure needs only $\left(\begin{array}{l}n \\ 1\end{array}\right)+\left(\begin{array}{l}n \\ 2\end{array}\right)+\cdots+\left(\begin{array}{l}n \\ k\end{array}\right)$ coefficients to be defined, instead of $2^{n}-1$.

Due to (3), an equivalent definition is: $\mu$ is $k$-additive if its interaction transform $I^{\mu}$ vanishes for subsets of more than $k$ elements, and there exists a subset $A$ of $k$ elements such that $I^{\mu}(A) \neq 0$. Since the interaction transform has a clear interpretation in the context of multicriteria decision making, $k$-additive fuzzy measures are of particular interest. Especially, 2-additive fuzzy measure have the advantage of being the simplest fuzzy measures (in terms of number of free coefficients) able to represent interaction between two elements.

$k$-additive fuzzy measures are families of fuzzy measures which are of polynomial complexity instead of the exponential complexity of general fuzzy measures. Another set of such families is provided by the concept of $p$-symmetric fuzzy measure (Miranda and Grabisch $[24,25])$. A fuzzy measure $\mu$ is symmetric if $\mu(A)=\mu(B)$ whenever $|A|=|B|$. Furthermore, two distinct elements $i, j \in X$ are symmetric w.r.t. a fuzzy measure $\mu$ (denoted by $\left.i \sim_{\mu} j\right)$ if $\mu(A \cup i)=\mu(A \cup j)$ for every $A \subseteq X \backslash\{i, j\}$. Note that $\sim_{\mu}$ is an 
equivalence relation, and let us consider its equivalence classes, which forms a partition of $X$. Clearly, a symmetric fuzzy measure has only one such equivalence class, which is $X$. A natural generalization is: a fuzzy measure is $p$-symmetric if $\sim_{\mu}$ has $p$ equivalence classes. It follows that any fuzzy measure is $p$-symmetric for some $1 \leq p \leq n$ (by the way, also $k$-additive for some $1 \leq k \leq n$ ).

Consider a $p$-symmetric fuzzy measure $\mu$, with set of equivalence classes $\left\{A_{1}, \ldots, A_{p}\right\}$, and a subset $B \subseteq X$. Clearly, the value $\mu(B)$ depends uniquely on the numbers $b_{1}, \ldots, b_{p}$, with $b_{i}:=\left|A_{i} \cap B\right|$. Since $0 \leqslant b_{i} \leqslant\left|A_{i}\right|$, it follows that $\mu$ needs $\prod_{i=1}^{p}\left(\left|A_{i}\right|+1\right)$ coefficients to be defined.

\subsection{Fuzzy measures on set systems}

A set system $\mathcal{F}$ on $X$ is a subcollection of $2^{X}$ containing $\varnothing$ and covering $X$, that is, $\bigcup_{A \in \mathcal{F}} A=X$. We consider in this section fuzzy measures whose domain is a set system.

We begin by introducing the main families of set systems of interest. The most classical example borrowed from measure theory is algebra. An algebra is a set system closed under finite union and complementation. Although complementation is fundamental in classical measure theory, this is no more the case for fuzzy measures and games, so that other algebraic structures arise:

(i) Set systems closed under union and intersection: (Faigle and Kern [26]) It follows that such set systems contain $X$ and are distributive lattices. Under the additional condition that there is no macro-element (i.e., a subset $M \subset X$ with $|M|>1$ such that for any $A \in \mathcal{F}$, either $M \subseteq A$ or $A \cap M=\varnothing)$, from Birkhoff's representation theorem, the set of all such set systems is in bijection with the set of partial orders on $X$. In other words, any such $\mathcal{F}$ is generated by a partial order on $X$, which can be interpreted as a kind of hierarchy of the elements in $X$. This is particularly meaningful when $X$ is a set of players, agents, etc., or criteria.

(ii) Weakly union-closed set systems: (Algaba [27], Faigle and Grabisch [28, 29]) $\mathcal{F}$ is weakly union-closed if $A, B \in \mathcal{F}, A \cap B \neq \varnothing$ imply $A \cup B \in \mathcal{F}$. This larger family is motivated by communication graphs. Suppose that a graph $(X, E)$ is defined on $X$, with $X$ being the set of nodes, and $E$ being the set of edges, i.e., pairs $\{i, j\}$ with $i, j \in X$ and $i \neq j$. Say that a subset $A \subseteq X$ is connected if for any distinct $i, j \in A$, there exists a sequence $i=i_{1}, i_{2}, \ldots, i_{q}=j$ of elements of $X$ such that $\left\{i_{k}, i_{k+1}\right\} \in E$ for $k=1, \ldots, q-1$. Defining $\mathcal{F}$ as the set of connected subsets of $X$, it follows that $\mathcal{F}$ is weakly union-closed (this is however not a characterizing property).

(iii) Regular set systems: $[30,31]$ a set system $\mathcal{F}$ is regular if it contains $X$ and any maximal chain ${ }^{1}$ from $\varnothing$ to $X$ has length $n$. Every distributive lattice is a regular set system. The motivation for such sets systems is more mathematical: it happens that many concepts around games and fuzzy measures are based on maximal chains of length $n$ (Shapley value, marginal vectors, Choquet integrals, etc.).

If $\mathcal{F}$ is a lattice (in particular, if $\mathcal{F}$ is closed under union and intersection), the definition of $k$-monotonicity is easily adapted by substituting $\cup, \cap$ in (1) by $\vee, \wedge$ of the lattice. It is well-known that when $\mathcal{F}=2^{X}$, there is an equivalence between total monotonicity

\footnotetext{
${ }^{1}$ A chain from $\varnothing$ to $X$ is a sequence $\varnothing=A_{0}, A_{1}, \ldots, A_{q}=X$ of sets in $\mathcal{F}$ such that $A_{0} \subset A_{1} \subset \cdots \subset A_{q}$. Its length is $q$, and the chain is maximal if no other chain from $\varnothing$ to $X$ contains it.
} 
and the nonnegativity of the Möbius transform. It has been for a long time an unsolved issue whether this equivalence still holds if $\mathcal{F}$ is a lattice, only recently solved:

Theorem 2. Let $\mu$ be fuzzy measure on a lattice $\mathcal{F}$. Then $\mu$ is totally monotone if and only if it has a nonnegative Möbius transform.

The "only if" part was shown by Barthélemy [32], and the "if part" recently by Zhou [33].

\section{The Choquet and Sugeno integrals}

The term "fuzzy integral" has been introduced by Sugeno [7] in 1974, and is now most commonly called the Sugeno integral. However, Choquet already in 1954 proposed a functional w.r.t. a fuzzy measure (or capacity), referred now as the Choquet integral. As we will see in Section 3.8, other integrals w.r.t. fuzzy measures have been proposed. We study in detail the Choquet and Sugeno integrals, which can be considered as the most representative (and still very different) fuzzy integrals. Except for Section 3.7, we assume that fuzzy measures are defined on $\mathcal{F}=2^{X}$.

\subsection{Definitions and basic properties}

We begin by introducing the general definition, which is valid for arbitrary spaces. For this, we need decumulative distribution functions. Let $\mu$ be a fuzzy measure and $f: X \rightarrow$ $\mathbb{R}$. The decumulative distribution of $f$ w.r.t. $\mu$ is

$$
G_{\mu, f}(t)=\mu(\{x \in X \mid f(x) \geqslant t\} \quad(t \in \mathbb{R}) .
$$

We consider first nonnegative functions. Let $f: X \rightarrow \mathbb{R}_{+}$and $\mu$ be a fuzzy measure. The Choquet integral of $f$ w.r.t. $\mu$ is defined by

$$
\int f \mathrm{~d} \mu=\int_{0}^{\infty} G_{\mu, f}(t) \mathrm{d} t
$$

where the right hand-side integral is the Riemann integral. The Sugeno integral of $f$ w.r.t. $\mu$ is defined by

$$
f f \mathrm{~d} \mu=\bigvee_{t \geqslant 0}\left(G_{\mu, f}(t) \wedge t\right)=\bigwedge_{t \geqslant 0}\left(G_{\mu, f}(t) \vee t\right) .
$$

In words, the Sugeno integral is the abscissa of the intersection point between the diagonal and the decumulative function, while the Choquet integral is the area below the decumulative function. It can be proven that it is equivalent to consider a strict inequality in the definition of $G_{\mu, f}$. Another equivalent formula for the Sugeno integral is

$$
f f \mathrm{~d} \mu=\bigvee_{A \in \mathcal{F}}\left(\bigwedge_{x \in A} f(x) \wedge \mu(A)\right)
$$

Note that the Choquet integral can be defined w.r.t. games as well. However, since the decumulative function is no more monotone with games, the definition of the Sugeno integral is restricted to fuzzy measures. An elementary property is that for every $A \subseteq X$, 
$\int 1_{A} \mathrm{~d} \mu=\mu(A)$, where $1_{A}$ is the characteristic function of $A$. The latter property holds also for the Sugeno integral, provided $\mu$ is normalized. In view of this property, the Choquet and Sugeno integrals can be considered as extensions of fuzzy measures.

When $X=\left\{x_{1}, \ldots, x_{n}\right\}$, the formulas can be made more explicit. For a function $f: X \rightarrow \mathbb{R}_{+}$, let $f_{i}$ denotes $f\left(x_{i}\right)$ for simplicity, and take a permutation $\sigma$ on $\{1, \ldots, n\}$ such that $f_{\sigma(1)} \leqslant \cdots \leqslant f_{\sigma(n)}$. Define $A_{\sigma}^{\uparrow}(i)=\left\{x_{\sigma(i)}, x_{\sigma(i+1)}, \ldots, x_{\sigma(n)}\right\}, i=1, \ldots, n$. The Choquet integral is given by

$$
\begin{aligned}
\int f \mathrm{~d} \mu & =\sum_{i=1}^{n}\left(f_{\sigma(i)}-f_{\sigma(i-1)}\right) \mu\left(A_{\sigma}^{\uparrow}(i)\right) \\
& =\sum_{i=1}^{n} f_{\sigma(i)}\left(\mu\left(A_{\sigma}^{\uparrow}(i)\right)-\mu\left(A_{\sigma}^{\uparrow}(i+1)\right)\right),
\end{aligned}
$$

with the conventions $f_{\sigma(0)}=0$ and $A_{\sigma}^{\uparrow}(n+1)=\varnothing$.

For the Sugeno integral, we obtain:

$$
\begin{aligned}
f f \mathrm{~d} \mu & =\bigvee_{i=1}^{n}\left(f_{\sigma(i)} \wedge \mu\left(A_{\sigma}^{\uparrow}(i)\right)\right) \\
& =\bigwedge_{i=0}^{n}\left(f_{\sigma(i)} \vee \mu\left(A_{\sigma}^{\uparrow}(i+1)\right)\right)
\end{aligned}
$$

with the same conventions.

We consider now the case of real-valued integrands. For any $f: X \rightarrow \mathbb{R}$, we write

$$
f=f^{+}-f^{-}, \text {with } f^{+}=0 \vee f, \quad f^{-}=(-f)^{+} .
$$

Then the symmetric Choquet integral (a.k.a. Šipoš integral [34]) is defined by

$$
\check{\int} f \mathrm{~d} \mu=\int f^{+} \mathrm{d} \mu-\int f^{-} \mathrm{d} \mu .
$$

The asymmetric Choquet integral, which is the usual definition, is defined by

$$
\int f \mathrm{~d} \mu=\int f^{+} \mathrm{d} \mu-\int f^{-} \mathrm{d} \bar{\mu}
$$

where $\bar{\mu}$ is the conjugate fuzzy measure, defined by $\bar{\mu}(A)=\mu(X)-\mu(X \backslash A)$ for any $A \in 2^{X}$. The asymmetric Choquet integral is translation invariant (it is the only extension having this property), while the symmetric integral satisfies

$$
\check{\int}(-f) \mathrm{d} \mu=-\check{\int} f \mathrm{~d} \mu \text {. }
$$

The case of the Sugeno integral is more cumbersome, essentially due to the following problem. The Sugeno integral is defined through the $\vee, \wedge$ operators, playing the rôle of addition and product respectively (compare (6) with (8)). Remembering that on the ring 
of real numbers, $a-b$ is shorthand for $a+(-b)$, a transposition of formula (10) for the Sugeno integral would read

$$
\check{f} f \mathrm{~d} \mu=f f^{+} \mathrm{d} \mu \otimes\left(-f f^{-} \mathrm{d} \mu\right)
$$

where $\otimes$ is an extension of $\vee$ for real numbers (i.e., $a \otimes b=a \vee b$ whenever $a, b \geqslant 0$ ) such that $a \otimes(-a)=0$. Surprisingly, such an operator $\otimes$ would be necessarily nonassociative. Indeed,

$$
\begin{gathered}
((-3) \oslash 3) \otimes 2)=0 \oslash 2=0 \vee 2=2 \\
(-3) \otimes(3 \otimes 2)=(-3) \oslash(3 \vee 2)=(-3) \oslash 3=0 .
\end{gathered}
$$

The lack of associativity forbids to infer the so-called rule of sign, i.e., $(-a) \otimes(-b)=$ $-(a \otimes b)$, which is necessary for the symmetry of the integral:

$$
\begin{aligned}
\check{f}(-f) \mathrm{d} \mu=f f^{-} \mathrm{d} \mu \otimes\left(-f f^{+} \mathrm{d} \mu\right)=-\left(\left(-f f^{-} \mathrm{d} \mu\right) \otimes f f^{+} \mathrm{d} \mu\right) & \\
& =-f f \mathrm{~d} \mu .
\end{aligned}
$$

It can be shown [35] that the best operator (in the sense that it is associative on the largest domain) satisfying the above requirements (including the rule of sign) is the symmetric maximum, defined by

$$
a \otimes b= \begin{cases}-(|a| \vee|b|), & \text { if } b \neq-a \text { and either }|a| \vee|b|=-a \text { or }=-b \\ 0, & \text { if } b=-a \\ |a| \vee|b|, & \text { otherwise. }\end{cases}
$$

The symmetric Sugeno integral [36] is therefore defined by (12) and $\emptyset$. Up to now, there is no adequate definition of an asymmetric Sugeno integral.

\subsection{The Choquet integral as a linear interpolator}

Consider the following problem: a function $I:[0,1]^{n} \rightarrow[0,1]$ is known only on the vertices of the hypercube $[0,1]^{n}$ (in particular $I(\mathbf{0})=0$, where $\mathbf{0}$ is the 0 vector), and has to be determined everywhere in the hypercube. This is an interpolation problem, and there exists many ways to make the interpolation. Noting that the vertices of the hypercube correspond bijectively to the subsets of $X$ (with $|X|=n$ ), it follows that $I$ is necessarily an extension of a game $v: I\left(1_{A}\right)=v(A)$ for every $A \in 2^{X}$. Hence the Choquet and Sugeno integrals could be candidate.

Even if we restrict to a linear interpolation, there are still many ways of doing the interpolation, depending on which vertices are chosen, but there exist two extreme ways. If all vertices are used for each point $f \in[0,1]^{n}$, we get the multilinear model (owen, citeowe88), given by:

$$
I(f)=\sum_{A \subseteq X, A \neq \text { varnothing }} m^{v}(A) \prod_{i \in A} f_{i}
$$


where $m^{v}$ is the Möbius transform of $v$, defined by $v(A)=I\left(1_{A}\right)$ for every $A \in 2^{X}$. The other extreme case would be to take the minimum number of vertices so that the considered vector $x$ is contained in the convex hull of the selected vertices (parsimonious interpolation). Then this number is $n+1$, the number of vertices of a $n$-dimensional simplex, and the problem of choosing the right simplices for each $f$ amounts to the triangulation problem of the hypercube. There is one triangulation of particular interest since it leads to an interpolation where all constant terms are 0 , the triangulation in the $n$ ! canonical simplices, where each simplex is induced by a permutation $\sigma$ on $\{1, \ldots, n\}$ :

$$
S_{\sigma}=\left\{f \in[0,1]^{n} \mid f_{\sigma(1)} \leqslant f_{\sigma(2)} \leqslant \cdots \leqslant f_{\sigma(n)}\right\} .
$$

Then it can be shown that the parsimonious linear interpolation based on the canonical simplices is the Choquet integral. This fact was remarked by Singer [37], and also Marichal $[38]$.

\subsection{Expression w.r.t transforms}

The Choquet integral being linear w.r.t. the game, it is easy to get its expression when the game is expressed by some linear invertible transform (equivalently, in some other basis). Let $\Psi$ be a linear invertible transform, and $\left\{b_{A}^{\Psi}\right\}_{A \in 2^{x}}$ the corresponding basis of set functions given by Lemma 1 . Since these set functions are not necessarily games, and the Choquet integral needs games to be well defined, we build a basis of games $\left\{b_{A}^{\prime \Psi}\right\}_{A \in 2^{X} \backslash\{\varnothing\}}$ as follows:

$$
b_{S}^{\prime}(T)= \begin{cases}b_{S}(T), & \text { if } T \neq \varnothing \quad\left(S \in 2^{X} \backslash\{\varnothing\}\right) . \\ 0, \text { otherwise } & \end{cases}
$$

Then for every $f \in \mathbb{R}^{X}$ and every game $v$,

$$
\int f \mathrm{~d} v=\int f \mathrm{~d}\left(\sum_{\varnothing \neq A \subseteq X} \Psi^{v}(A) b_{A}^{\prime \Psi}\right)=\sum_{\varnothing \neq A \subseteq X} \Psi^{v}(A) \int f \mathrm{~d} b_{A}^{\prime \Psi} .
$$

It is therefore sufficient to compute $\int f \mathrm{~d} b_{A}^{\prime \Psi}$ for every $A \subseteq X, A \neq \varnothing$.

Applying this to the Möbius transform immedaitely yields the following well-known formula:

$$
\int f \mathrm{~d} v=\sum_{A \subseteq X} m^{v}(A) \bigwedge_{i \in A} f_{i}
$$

The same methodology is not applicable to the Sugeno integral since it is not linear w.r.t. the fuzzy measure. It is possible however to obtain a formula similar to (17), by means of the ordinal Möbius transform. The ordinal Möbius transform of a fuzzy measure $\mu$ is the interval $[m]:=\left[m_{*}, m^{*}\right]$, with $m^{*}=\mu$, and

$$
m_{*}(A)=\left\{\begin{array}{ll}
\mu(A), & \text { if } \mu(A)>\mu(A \backslash i), \forall i \in A \\
0, & \text { otherwise }
\end{array} \quad(A \subseteq X) .\right.
$$

The above formula has been first proposed in [39] [40], then developed in [35]. Then, it can be proved that the Sugeno integral takes the form:

$$
f f \mathrm{~d} \mu=\bigvee_{A \subseteq X}\left(\bigwedge_{i \in A} f_{i} \wedge m(A)\right)
$$

where $m$ is any function in $\left[m_{*}, m^{*}\right]$. 


\subsection{Properties}

The next propositions summarize the main elementary properties of Choquet and Sugeno integrals. In the whole section, $X$ is supposed to be finite, and $\mathcal{F}=2^{X}$.

Theorem 3. Let $f: X \rightarrow \mathbb{R}$ be a function and a game $v$. The following properties hold for the Choquet integral.

(i) Positive homogeneity:

$$
\int \alpha f \mathrm{~d} v=\alpha \int f \mathrm{~d} v \quad(\alpha \geqslant 0)
$$

(ii) Homogeneity of the symmetric Choquet integral:

$$
\check{\int} \alpha f \mathrm{~d} v=\alpha \check{\int} f \mathrm{~d} v \quad(\alpha \in \mathbb{R})
$$

(iii) Translation invariance:

$$
\int\left(f+\alpha 1_{X}\right) \mathrm{d} v=\int f \mathrm{~d} v+\alpha v(X) \quad(\alpha \in \mathbb{R})
$$

(iv) Asymmetry:

$$
\int(-f) \mathrm{d} v=-\int f \mathrm{~d} \bar{v}
$$

where $\bar{v}$ is the conjugate game;

(v) Scale inversion:

$$
\int\left(\alpha 1_{X}-f\right) \mathrm{d} v=\alpha v(X)-\int f \mathrm{~d} \bar{v} \quad(\alpha \in \mathbb{R})
$$

(vi) Monotonicity w.r.t. the integrand: for any fuzzy measure $\mu$,

$$
f \leqslant f^{\prime} \Rightarrow \int f \mathrm{~d} \mu \leqslant \int f^{\prime} \mathrm{d} \mu
$$

(vii) Monotonicity w.r.t. the game for nonnegative integrands: if $f \geqslant 0$,

$$
v \leqslant v^{\prime} \Rightarrow \int f \mathrm{~d} v \leq \int f \mathrm{~d} v^{\prime}
$$

(viii) Linearity w.r.t. the game:

$$
\int f \mathrm{~d}\left(v+\alpha v^{\prime}\right)=\int f \mathrm{~d} v+\alpha \int f \mathrm{~d} v^{\prime}, \quad(\alpha \in \mathbb{R})
$$

(ix) Boundaries: inf $f$ and $\sup f$ are attained:

$$
\inf f=\int f \mathrm{~d} \mu_{\min }, \quad \sup f=\int f \mathrm{~d} \mu_{\max },
$$

with $\mu_{\min }(A)=0$ for all $A \subset X$, and $\mu_{\max }(A)=1$ for all nonempty $A \subseteq X$; 
(x) Continuity.

Theorem 4. Let $f: X \rightarrow \mathbb{R}_{+}$, and $\mu$ a fuzzy measure on $X$. The following properties hold for the Sugeno integral.

(i) Positive ^-homogeneity:

$$
f\left(\alpha 1_{X} \wedge f\right) \mathrm{d} \mu=\alpha \wedge f f \mathrm{~d} \mu \quad(\alpha \geqslant 0)
$$

(ii) Positive $\vee$-homogeneity if $\sup f \leqslant \mu(X)$ :

$$
f\left(\alpha 1_{X} \vee f\right) \mathrm{d} \mu=\alpha \vee f f \mathrm{~d} \mu \quad(\alpha \in[0, \sup f]) .
$$

(iii) Hat function: for every $\alpha \geqslant 0$ and for every $A \in \mathcal{F}$,

$$
f \alpha 1_{A} \mathrm{~d} \mu=\alpha \wedge \mu(A)
$$

(iv) Scale inversion: if $\sup f \leqslant \mu(X)$,

$$
f\left(\mu(X) 1_{X}-f\right) \mathrm{d} \mu=\mu(X)-f f \mathrm{~d} \bar{\mu},
$$

where $\bar{\mu}$ is the conjugate fuzzy measure;

(v) Scale translation:

$$
f\left(f+\alpha 1_{X}\right) \mathrm{d} \mu \leqslant f f \mathrm{~d} \mu+f \alpha \mathrm{d} \mu=f f \mathrm{~d} \mu+\alpha \wedge \mu(X) \quad(\alpha \geqslant 0)
$$

(vi) Monotonicity w.r.t. the integrand:

$$
f \leqslant f^{\prime} \Rightarrow f f \mathrm{~d} \mu \leqslant f f^{\prime} \mathrm{d} \mu \quad\left(f, f^{\prime} \in B^{+}(\mathcal{F})\right)
$$

(vii) Monotonicity w.r.t. the fuzzy measure:

$$
\mu \leqslant \mu^{\prime} \Rightarrow f f \mathrm{~d} \mu \leq f f \mathrm{~d} \mu^{\prime}
$$

(viii) Max-min linearity w.r.t. the fuzzy measure:

$$
f f \mathrm{~d}\left(\mu \vee\left(\alpha \wedge \mu^{\prime}\right)\right)=f f \mathrm{~d} \mu \vee\left(\alpha \wedge \int f \mathrm{~d} \mu^{\prime}\right) \quad(\alpha \geqslant 0)
$$

(ix) Boundaries: $\inf f$ and $\sup f$ are attained:

$$
\inf f=f f \mathrm{~d} \mu_{\min }, \quad \sup f=f f \mathrm{~d} \mu_{\max },
$$

with $\mu_{\min }, \mu_{\max }$ defined as in Theorem 3; 
(x) Lipschitz continuity:

$$
|f f \mathrm{~d} \mu-f g \mathrm{~d} \mu| \leqslant \mu(X) \wedge\|f-g\| \quad\left(f, g \in B^{+}(\mathcal{F})\right)
$$

with $\|f\|=\sup _{x \in X}|f(x)|$ (Chebyshev norm). Hence, if $\mu$ is normalized and $f, g$ are valued on $[0,1]$, we obtain that the Sugeno integral is 1-Lipschitzian for the Chebyshev norm.

A fundamental feature of both Choquet and Sugeno integrals is their relation with comonotonic functions. Two functions $f, g: X \rightarrow \mathbb{R}$ are comonotonic if there is no $x, x^{\prime} \in$ $X$ such that $f(x)<f\left(x^{\prime}\right)$ and $g(x)>g\left(x^{\prime}\right)$ (equivalently, in the case of a finite universe, if there exists a permutation $\sigma$ on $X$ such that $f_{\sigma(1)} \leqslant \cdots \leqslant f_{\sigma(n)}$ and $\left.g_{\sigma(1)} \leqslant \cdots \leqslant g_{\sigma(n)}\right)$.

Theorem 5. Let $f, g$ be comonotonic functions on $X$ (finite). Then for any game $v$, the Choquet integral is comonotonically additive, and the Sugeno integral is comonotonically maxitive and minitive for any fuzzy measure $\mu$ :

$$
\begin{aligned}
& \int(f+g) \mathrm{d} v=\int f \mathrm{~d} v+\int g \mathrm{~d} v \\
& f(f \vee g) \mathrm{d} \mu=f f \mathrm{~d} \mu \vee f g \mathrm{~d} \mu \\
& f(f \wedge g) \mathrm{d} \mu=f f \mathrm{~d} \mu \wedge f g \mathrm{~d} \mu .
\end{aligned}
$$

A more recently introduced type of additivity is called horizontal additivity (see Šipoš [34], and Benvenuti et al. [41]). Given a function $f: X \rightarrow \mathbb{R}$ and a constant $c \in \mathbb{R}$, the horizontal min-additive decomposition of $f$ is:

$$
f=\left(f \wedge c 1_{X}\right)+\left(f-\left(f \wedge c 1_{X}\right)\right) .
$$

This amounts to "cut" horizontally the function at level $c$. Similarly, the horizontal maxadditive decomposition of $f$ is:

$$
f=\left(f \vee c 1_{X}\right)+\left(f-\left(f \vee c 1_{X}\right)\right) .
$$

A functional $I: \mathbb{R}^{X} \rightarrow \mathbb{R}$ is horizontally min-additive if for every $f: X \rightarrow \mathbb{R}$ and $c \in \mathbb{R}$,

$$
I(f)=I\left(f \wedge c 1_{X}\right)+I\left(f-\left(f \wedge c 1_{X}\right)\right) .
$$

Horizontal max-additivity is defined similarly. It turns out that these notions are equivalent to comonotonic additivity, as shown by Couceiro and Marichal [42]. A related notion is horizontal median-additivity, introduced by Couceiro and Marichal [42]. Lastly, we introduce comonotonic modularity. A functional $I: \mathbb{R}^{X} \rightarrow \mathbb{R}$ is modular if for every $f, g: X \rightarrow \mathbb{R}$

$$
I(f \vee g)+I(f \wedge g)=I(f)+I(g) .
$$

It can be easily shown that the Choquet integral is comonotonically modular, i.e., for any comonotonic functions $f, g$ it holds

$$
\int(f \vee g) \mathrm{d} v+\int(f \wedge g) \mathrm{d} v=\int f \mathrm{~d} v+\int g \mathrm{~d} v
$$


This also holds for the Sugeno integral.

The next theorem clarifies the important case of supermodular fuzzy measures for the Choquet integral.

Theorem 6. For any game v, the following conditions are equivalent:

(i) $v$ is supermodular;

(ii) The Choquet integral is superadditive, that is,

$$
\int(f+g) \mathrm{d} v \geqslant \int f \mathrm{~d} v+\int g \mathrm{~d} v
$$

for all $f, g: X \rightarrow \mathbb{R}$

(iii) The Choquet integral is supermodular, that is,

$$
\int(f \vee g) \mathrm{d} v+\int(f \wedge g) \mathrm{d} v \geqslant \int f \mathrm{~d} v+\int g \mathrm{~d} v
$$

for all $f, g: X \rightarrow \mathbb{R}$

(iv) The Choquet integral is concave, that is,

$$
\int(\lambda f+(1-\lambda) g) \mathrm{d} v \geqslant \int \lambda f \mathrm{~d} v+(1-\lambda) \int g \mathrm{~d} v
$$

for all $\lambda \in[0,1], f, g: X \rightarrow \mathbb{R}$.

(v) The Choquet integral yields the lower expected value onthe core of $v$ :

$$
\int f \mathrm{~d} v=\min _{\phi \in \operatorname{core}(v)} \int f \mathrm{~d} \phi,
$$

where core $(v)$ is the set of additive games $\phi$ on $X$ such that $\phi(X)=v(X)$ and $\phi(S) \geqslant$ $v(S)$ for all $S \in 2^{X}$.

Lastly, we give the properties of the Sugeno integral concerning maxitivity and minitivity.

Theorem 7. The following holds:

(i) $f(f \vee g) \mathrm{d} \mu=f f \mathrm{~d} \mu \vee f g \mathrm{~d} \mu$ for all $f, g \in B^{+}(\mathcal{F})$ if and only if $\mu$ is maxitive;

(ii) $f(f \wedge g) \mathrm{d} \mu=f f \mathrm{~d} \mu \wedge f g \mathrm{~d} \mu$ for all $f, g \in B^{+}(\mathcal{F})$ if and only if $\mu$ is minitive.

\subsection{Characterizations}

The most famous characterization of the Choquet integral is due to Schmeider [43], whose adaptation to the finite case $(|X|=n)$ and $\mathcal{F}=2^{X}$ is as follows.

Theorem 8. Let $I: \mathbb{R}^{X} \rightarrow \mathbb{R}$ be a functional. Define the set function $v(A)=I\left(1_{A}\right)$ on $2^{X}$. The following propositions are equivalent:

(i) I is monotone and comonotonically additive;

(ii) $v$ is a fuzzy measure, and for all $f \in \mathbb{R}^{N}, I(f)=\int f \mathrm{~d} v$. 
The discrete version (with a redundant axiom) was shown by de Campos and Bolaños [44]. A similar characterization for the Choquet integral w.r.t. games was obtained by Murofushi et al. [45].

In the discrete case, a characterization using comonotonic modularity was obtained by Couceiro and Marichal [46, 47].

Theorem 9. Let $|X|=n$ and $\mathcal{F}=2^{X}$, and let $I: \mathbb{R}^{X} \rightarrow \mathbb{R}$ be a functional. Define the set function $v(A)=I\left(1_{A}\right), A \subseteq X$. The following propositions are equivalent:

(i) $I$ is comonotonically modular and satisfies $I\left(\alpha 1_{S}\right)=|\alpha| I\left(\operatorname{sign}(\alpha) 1_{S}\right)$ for all $\alpha \in \mathbb{R}$ and $S \subseteq X$, and $I\left(1_{X \backslash S}\right)=I\left(1_{X}\right)+I\left(-1_{S}\right)$;

(ii) $v$ is a game and $I(f)=\int f \mathrm{~d} v$.

The Sugeno integral was characterized in the discrete case by de Campos and Bolaños [44]. Here follows a simplified and more general version.

Theorem 10. Let $|X|=n, \mathcal{F}=2^{X}$, and let $I:\left(\mathbb{R}_{+}\right)^{X} \rightarrow \mathbb{R}_{+}$be a functional. Define the set function $\mu(A)=I\left(1_{A}\right), A \subseteq X$. The following propositions are equivalent:

(i) $I$ is comonotonically maxitive, satisfies $I\left(\alpha 1_{A}\right)=\alpha \wedge I\left(1_{A}\right)$ for every $\alpha \geqslant 0$ and $A \subseteq X$, and $I\left(1_{X}\right)=1$;

(ii) $\mu$ is a normalized fuzzy measure on $X$ and $I(f)=f f \mathrm{~d} \mu$.

The next characterization is due to Marichal [48]. Still others can be found in this reference.

Theorem 11. Let $|X|=n, \mathcal{F}=2^{X}$, and let $I:[0,1]^{X} \rightarrow[0,1]$ be a functional. Define the set function $\mu(A)=I\left(1_{A}\right), A \subseteq X$. The following propositions are equivalent:

(i) I is nondecreasing, $\vee$-homogeneous and $\wedge$-homogeneous;

(ii) $\mu$ is a normalized fuzzy measure on $X$ and $I(f)=f f \mathrm{~d} \mu$.

\subsection{The Choquet integral on the nonnegative real line}

As remarked by Sugeno in two recent papers [49,50], so far there is no "Choquet integral calculus", similar to classical integral calculus, even if one restricts to functions and measures on the real line. By means of the Laplace transform, Sugeno established in these two papers the basis of Choquet integral calculus. For this, the Choquet integral on a restricted domain is used:

$$
\int_{A} f \mathrm{~d} \mu=\int_{0}^{\infty} \mu(\{x \geqslant t\} \cap A) \mathrm{d} t
$$

for some $A \subseteq X$. We give now the fundamental theorem.

Theorem 12. Let $f: \mathbb{R}_{+} \rightarrow \mathbb{R}_{+}$be nondecreasing and continuously differentiable, and let $\mu$ be a continuous fuzzy measure on $\mathbb{R}_{+}$, such that $\mu([\tau, t])$ is differentiable w.r.t. $\tau$ on $[0, t]$ for every $t>0$, and $\mu(\{t\})=0$ for every $t \geqslant 0$. Then

$$
\int_{[0, t]} f \mathrm{~d} \mu=-\int_{0}^{t} \frac{\partial \mu}{\partial \tau}([\tau, t]) f(\tau) \mathrm{d} \tau \quad(t>0),
$$


where the righthand side integral is the Riemann integral. In particular, for a distorted Lebesgue measure $\mu_{h}$ with $h$ being continuously differentiable, we obtain

$$
\int_{[0, t]} f \mathrm{~d} \mu_{h}=\int_{0}^{t} \frac{\partial h}{\partial \tau}(t-\tau) f(\tau) \mathrm{d} \tau
$$

Equation (21 can be computed very easily through the Laplace transform. Denoting by $\mathcal{L}^{-1}$ the inverse Laplace transform, and by $H(s)$ and $F(s)$ the Laplace transforms of $h$ and $f$, we have:

$$
\int_{[0, t]} f \mathrm{~d} \mu_{h}=\mathcal{L}^{-1}(s H(s) F(s)) .
$$

\subsection{The Choquet integral of nonmeasurable functions}

So far we have considered that $\mathcal{F}=2^{X}$, so that every subset is measurable and consequently any function is measurable too (i.e., its level sets belong to $\mathcal{F}$ ). In the case where $\mathcal{F} \subset 2^{X}$, what about the integral of a nonmeasurable function? The question may appear quite odd, but makes sense in practical situations, for example in multicriteria decision making. In this field, $X$ is the set of criteria and $\mu(A)$ for some $A \subseteq X$ is interpreted as the overall evaluation of an alternative being satisfactory on criteria in $A$, and unsatisfactory or neutral on the others. It may be the case that such an alternative is not conceivable, and so no value can be assigned to $\mu(A)$. However, when computing the overall score of an alternative, knowing the vector $f$ of its scores on every criterion, the set $A$ may be a level set of $f$ (i.e., $A=\{x \in X \mid f(x) \geqslant t\}$ for some $t$ ), so that $f$ is not measurable and its Choquet integral cannot be computed. In this section we indicate how to extend the Choquet integral to nonmeasurable functions. This work is based on [28].

Let $\mathcal{F}$ be a fixed set system. We decompose any game $v$ on $\mathcal{F}$ as $v=v^{+}+v^{-}$, where $v^{+}, v^{-}$are two totally monotone fuzzy measures:

$$
v^{+}=\sum_{A \in \mathcal{F} \mid m^{v}(A)>0} m^{v}(A) u_{A}, \quad v^{-}=\sum_{A \in \mathcal{F} \mid m^{v}(A)<0}\left(-m^{v}(A)\right) u_{A} .
$$

We first define the Choquet integral w.r.t. a totally monotone fuzzy measure $b$ on $\mathcal{F}$ as follows ( $f$ is assumed to be nonnegative):

$$
\begin{aligned}
\int_{\mathcal{F}} f \mathrm{~d} b & =\max \left\{\sum_{A \in \mathcal{F}} \alpha_{A} b(A) \mid \sum_{A \in \mathcal{F}} \alpha_{A} 1_{A} \leqslant f, \alpha_{A} \geqslant 0, \forall A \in \mathcal{F}\right\} \\
& =\min \left\{\sum_{i \in X} P_{i} f_{i} \mid \sum_{i \in A} P_{i} \geqslant b(A), \forall A \in \mathcal{F}, P_{i} \geqslant 0, \forall i \in X\right\} .
\end{aligned}
$$

It can be proved that this is the smallest functional $I$ satisfying positive homogeneity, superadditivity and $I\left(1_{A}\right) \geqslant b(A)$ for all $A \in \mathcal{F}$. Now, the Choquet integral for any function $f: X \rightarrow \mathbb{R}$ w.r.t. a game $v$ is defined by

$$
\int_{\mathcal{F}} f \mathrm{~d} v=\int_{\mathcal{F}} f \mathrm{~d} v^{+}-\int_{\mathcal{F}} f \mathrm{~d} v^{-}
$$

We summarize the main properties of this integral. 
Theorem 13. Let $f: X \rightarrow \mathbb{R}_{+}$be a function and $v$ be a game on $(X, \mathcal{F})$, where $\mathcal{F}$ is any set system. The following properties hold.

(i) Positive homogeneity:

$$
\int_{\mathcal{F}} \alpha f \mathrm{~d} v=\alpha \int_{\mathcal{F}} f \mathrm{~d} v \quad(\alpha \geqslant 0)
$$

(ii) For any $S \in \mathcal{F}$,

$$
\int_{\mathcal{F}} f \mathrm{~d} u_{S}=\min _{i \in S} f_{i}
$$

where $u_{S}$ is the unanimity game w.r.t. $S$;

(iii) If $\mathcal{F}$ is weakly union-closed,

$$
\int_{\mathcal{F}} f \mathrm{~d} v=\sum_{S \in \mathcal{F}} m^{v}(S) \min _{i \in S} f_{i}
$$

where $m^{v}$ is the Möbius transform of $v$;

(iv) If $\mathcal{F}$ is weakly union-closed,

$$
\int_{\mathcal{F}} f \mathrm{~d} v=\int f \mathrm{~d} \hat{v}
$$

where the right-hand side integral is the ordinary Choquet integral, and $\hat{v}$ is a game on $\left(X, 2^{X}\right)$ defined by

$$
\hat{v}(S)=\int_{\mathcal{F}} 1_{S} \mathrm{~d} v=\sum_{F \text { maximum in } \mathcal{F}(S)} v(F) \quad\left(S \in 2^{X}\right),
$$

with $\mathcal{F}(S)=\{F \in \mathcal{F} \mid F \subseteq S\}$.

(v) If $\mathcal{F}$ is weakly union-closed, $\int_{\mathcal{F}} \cdot \mathrm{d} v$ is superadditive if and only if it is concave if and only if $\hat{v}$ is supermodular.

From (iv) we see that this integral is essentially the Choquet integral w.r.t. a modified game $\hat{v}$, and therefore inherits all of its properties. Moreover, $\hat{v}$ is an extension of $v$ in the sense that it coincides with $v$ on $\mathcal{F}$. It turns out that this integral yields the Choquet integral for measurable functions, and is indeed an extension of the Choquet integral. Note however that if $v$ is monotone, $\hat{v}$ is not necessarily so.

More results can be obtained if $\mathcal{F}$ is closed under union. In this case, it can be shown that a fuzzy measure $\mu$ on $\mathcal{F}$ is supermodular if and only if $\hat{\mu}$ is, where supermodularity for $\mu$ is defined as follows: for any $S, T \in \mathcal{F}$,

$$
\mu(S \cup T)+\mu\left((S \cap T)^{\prime}\right) \geqslant \mu(S)+\mu(T)
$$

where $(S \cap T)^{\prime}$ is the largest subset of $S \cap T$ in $\mathcal{F}$. Moreover, the following holds.

Theorem 14. Let $\mathcal{F}$ be a set system closed under union, and $\mu$ be a fuzzy measure on $(X, \mathcal{F})$. The following are equivalent: 
(i) For every function $f: X \rightarrow \mathbb{R}_{+}$,

$$
\begin{aligned}
\int_{\mathcal{F}} f \mathrm{~d} \mu & =\max \left\{\sum_{S \in \mathcal{F}} \lambda_{S} \mu(S) \mid \sum_{S \in \mathcal{F}} \lambda_{S} 1_{S} \leqslant f, \lambda \geqslant \mathbf{0}\right\} \\
& =\min \left\{\sum_{i \in X} P_{i} f_{i} \mid P(S) \geqslant \mu(S), \forall S \in \mathcal{F}, P \geqslant \mathbf{0}\right\},
\end{aligned}
$$

where $\mathbf{0}$ indicates the 0 vector.

(ii) $\int_{\mathcal{F}} \cdot \mathrm{d} \mu$ is superadditive;

(iii) $\mu$ is supermodular.

\subsection{Other integrals}

We describe briefly other kinds of integrals defined with respect to fuzzy measures.

Pseudo-additive integrals and fuzzy t-conorm integrals It is possible to define other integrals by simply replacing the operations used in the definitions of Choquet and Sugeno integrals (sum, product, max, min) by other ones, generally speaking, by pseudo-additions and pseudo-multiplications. There has been many studies in this direction, starting from Weber [51] and Kruse [52], then later Sugeno and Murofushi [53], Murofushi and Sugeno (fuzzy t-conorm integral) [54], Klement, Mesiar and Pap ((S,U)-integral) [55], Benvenuti et al. [41], and more recently the impressive study by Sander and Siedekum [56-58].

Basically, the $(S, U)$-integral uses as basis operators a continuous t-conorm $S$ and a uninorm $U$ which is distributive w.r.t. $S$ in the following sense:

$$
U(x, S(y, z))=S(U(x, y), U(x, z))
$$

for all $x, y, z \in[0,1]$ such that $S(y, z)<1$.

The fuzzy t-conorm integral proposed by Murofushi and Sugeno uses three continuous t-conorms $S_{1}, S_{2}, S_{3}$ which are either the maximum or Archimedean, plus a pseudomultiplication $\odot$, being nondecreasing in each place, continuous on $] 0,1]^{2}$, and satisfying $a \odot x=0$ implies either $a=0$ or $x=0$, and two distributivity properties:

(D1) $S_{1}(a, b)<1$ implies $\left(S_{1}(a, b)\right) \odot x=S_{3}((a \odot x),(b \odot x))$

(D2) $S_{2}(x, y)<1$ implies $a \odot\left(S_{2}(x, y)\right)=S_{3}((a \odot x),(a \odot y))$.

The definition of the fuzzy t-conorm integral is then:

$$
\left(S_{1}, S_{2}, S_{3}, \odot\right) \int f \mathrm{~d} \mu:=\stackrel{n}{\mathrm{~S}}_{3}\left(f_{\sigma(i)} \stackrel{S_{1}}{-} f_{\sigma(i-1)}\right) \odot \mu\left(A_{\sigma(i)}\right)
$$

with same notation as above, and $\stackrel{S_{1}}{-}$ is the residuated difference w.r.t. $S_{1}$, defined by

$$
a-b:=\inf \left\{c \mid S_{1}(b, c) \geqslant a\right\}
$$

for any $(a, b)$ in $[0,1]^{2}$. The Choquet integral is recovered with $S_{1}, S-2, S_{3}$ being the Łukasiewicz t-conorm, and $\odot$ the usual product. The Sugeno integral is recovered with $S_{1}=S_{2}=S_{3}=\max$ and $\odot=\min$, and the Shilkret [59] integral is obtained when $\odot$ is the ordinary product.

The integral proposed by Benvenuti et al. is similar. 
Universal integrals Universal integrals, proposed by Klement et al. [60] (see also a more recent work [61]), try to answer the following question: What is an integral w.r.t. a fuzzy measure? The answer given by Klement et al. is axiomatic: they propose a list of axioms a functional should satisfy to be considered as a integral. The name "universal" comes from the fact that the integral should be defined for any measurable space $(X, \mathcal{A})$ where $\mathcal{A}$ is a $\sigma$-algebra.

They first define a pseudo-multiplication as an operator $\otimes:[0, \infty]^{2} \rightarrow[0, \infty]$ satisfying the following properties: it is nondecreasing in each place, 0 is an annihilator of $\otimes$, i.e., $a \otimes 0=0 \otimes a=0$, and $\otimes$ has a neutral element $e \neq 0$, i.e., $a \otimes e=e \otimes a=a$.

Let us denote by $\mathcal{D}$ the set of all Cartesian products $\mathcal{M}(X, \mathcal{A}) \times \mathcal{F}(X, \mathcal{A})$ for every measurable space $(X, \mathcal{A})$, where $\mathcal{M}(X, \mathcal{A})$ is the set of fuzzy measures on $(X, \mathcal{A})$, and $\mathcal{F}(X, \mathcal{A})$ is the set of $\mathcal{A}$-measurable functions. A functional $I: \mathcal{D} \rightarrow[0, \infty]$ is called a universal integral of it satisfies the three following axioms:

(i) For any measurable space $(X, \mathcal{A})$, its restriction to $\mathcal{M}(X, \mathcal{A}) \times \mathcal{F}(X, \mathcal{A})$ is nondecreasing in each place

(ii) There exists a pseudo-multiplication $\otimes$ such that for all $\left(\mu, c \cdot 1_{A}\right) \in \mathcal{D}, I\left(\mu, c \cdot 1_{A}\right)=$ $c \otimes \mu(A)$

(iii) $I(\mu, f)=I\left(\mu^{\prime}, f^{\prime}\right)$ if $G_{\mu, f}=G_{\mu^{\prime}, f^{\prime}}$.

Obviously, the Choquet integral and the Sugeno integrals are universal integrals. It is not difficult to see that a universal integral is a distortion of the decumulative function by a function $J$ begin nondecreasing and satisfying $J\left(d \cdot 1_{0, c]}=c \otimes d\right.$. The Sugeno and Shilkret integrals belong to the set of smallest universal integrals (in the sense of the usual partial order on functions), given by

$$
I_{\otimes}(\mu, f)=\sup _{t \in] 0, \text { infty] }}\left(t \otimes G_{\mu, f}(t)\right) .
$$

It can be shown that all integrals of the form (5), with product and addition being replaced by a pseudo-multiplication $\otimes$ and a pseudo-addition $\oplus$ being continuous, associative, nondecreasing, having 0 as neutral element and being left-distributive w.r.t. $\otimes$, are universal integrals.

The concave integral and decomposition integral Recenty, in a series of papers Lehrer presented the concave integral [62-64], and a more general concept called the decomposition integral [65], encompassing both the concave integral and the Choquet integral, as well as the Shilkret integral.

We first introduce the concave integral. Let $f: X \rightarrow \mathbb{R}_{+}$and $\mu$ be a fuzzy measure. The concave integral of $f$ w.r.t. $\mu$ is given by:

$$
\int^{\text {cav }} f \mathrm{~d} \mu=\sup \left\{\sum_{S \subseteq X} \alpha_{S} \mu(S) \mid \sum_{S \subseteq X} \alpha_{S} 1_{S}=f, \quad \alpha_{S} \geqslant 0, \forall S \subseteq X\right\} .
$$

In words, the concave integral is the value achieved by the best decomposition of the integrand into hat functions. Note that for totally monotone fuzzy measures, the concave integral and the integral proposed by Faigle and Grabisch coincide (see Section 3.7).

Its main properties are given below. 
Theorem 15. The following properties hold for the concave integral:

(i) For every fuzzy measure $\mu$, the concave integral $\int^{\mathrm{cav}} \cdot \mathrm{d} \mu$ is a concave and positively homogeneous functional, and satisfies $\int^{\text {cav }} 1_{S} \mathrm{~d} \mu \geqslant \mu(S)$ for all $S \in 2^{X}$;

(ii) For every $f \in \mathbb{R}_{+}^{X}$ and fuzzy measure $\mu$,

$$
\begin{array}{r}
\int^{\text {cav }} f \mathrm{~d} \mu=\min \left\{I(f) \mid I: \mathbb{R}_{+}^{X} \rightarrow \mathbb{R}\right. \text { concave, positively homogeneous, } \\
\text { and such that } \left.I\left(1_{S}\right) \geqslant \mu(S), \forall S \subseteq X\right\}
\end{array}
$$

(iii) For every $f \in \mathbb{R}_{+}^{X}$ and fuzzy measure $\mu$,

$$
\int^{\text {cav }} f \mathrm{~d} \mu=\min _{P \text { additive }, P \geqslant \mu} \int f \mathrm{~d} P
$$

(iv) For every $f \in \mathbb{R}_{+}^{X}$ and fuzzy measure $\mu$,

$$
\int f \mathrm{~d} \mu \leqslant \int^{\mathrm{cav}} f \mathrm{~d} \mu
$$

and equality holds for every $f \in \mathbb{R}_{+}^{X}$ if and only if $\mu$ is supermodular.

Property (iv) clearly shows that unless the fuzzy measure is supermodular, the Choquet integral and the concave integral differ.

As for the decomposition integral, the idea is simply to fix a "vocabulary" for the decompositions. If only chains are allowed for the decomposition of a function, then the Choquet integral obtains as the best achievable value for such decompositions. If no restriction applies, then the concave integral is obtained. Also, the Shilkret integral can also be recovered. We refer the reader to [65] for full details on this complex notion.

\section{References}

1. E. Pap. Null-Additive Set Functions. Kluwer Academic, 1995.

2. D. Denneberg. Non-Additive Measure and Integral. Kluwer Academic, 1994.

3. Z. Wang and G. J. Klir. Generalized measure theory. Springer, 2009.

4. E. Pap, editor. Handbook of measure theory. Elsevier, 2002.

5. M. Grabisch, T. Murofushi, and M. Sugeno. Fuzzy Measures and Integrals. Theory and Applications (edited volume). Studies in Fuzziness. Physica Verlag, 2000.

6. M. Grabisch and Ch. Labreuche. A decade of application of the Choquet and Sugeno integrals in multi-criteria decision aid. Annals of Operations Research, 175:247-286, 2010. doi: 10.1007/s10479-009-0655-8.

7. M. Sugeno. Theory of fuzzy integrals and its applications. PhD thesis, Tokyo Institute of Technology, 1974.

8. G. Choquet. Theory of capacities. Annales de l'Institut Fourier, 5:131-295, 1953.

9. L. A. Zadeh. Fuzzy sets as a basis for a theory of possibility. Fuzzy Sets Es Systems, 1:3-28, 1978.

10. D. Dubois and H. Prade. Possibility Theory. Plenum Press, 1988.

11. G. Shafer. A Mathematical Theory of Evidence. Princeton Univ. Press, 1976.

12. R. Stanley. Two poset polytopes. Discrete and Computational Geometry, 1:9-23, 1986.

13. D. Radojevic. The logical representation of the discrete Choquet integral. Belgian Journal of Operations Research, Statistics and Computer Science, 38:67-89, 1998.

14. U. Faigle and M. Grabisch. Linear transforms, values and least square approximation for cooperation systems. working paper, 2014.

15. M. Grabisch. $k$-order additive discrete fuzzy measures and their representation. Fuzzy Sets and Systems, 92:167-189, 1997. 
16. L. S. Shapley. A value for $n$-person games. In H. W. Kuhn and A. W. Tucker, editors, Contributions to the Theory of Games, Vol. II, number 28 in Annals of Mathematics Studies, pages 307-317. Princeton University Press, 1953.

17. T. Murofushi and S. Soneda. Techniques for reading fuzzy measures (III): interaction index. In 9th Fuzzy System Symposium, pages 693-696, Sapporo, Japan, May 1993. In Japanese.

18. M. Grabisch. The application of fuzzy integrals in multicriteria decision making. European J. of Operational Research, 89:445-456, 1996.

19. R. de Wolf. A brief introduction to Fourier analysis on the Boolean cube. Theory of Computing Library Graduate Surveys, 1:1-20, 2008.

20. R. O'Donnell. Analysis of Boolean functions, draft 2.0, ch. 1-3. http://www.cs.cmu.edu/ odonnell11/boolean-analysis, 2007.

21. S. Hurst, D. Miller, and J. Muzio. Spectral techniques in digital logic. Academic Press, London, 1985.

22. M. Grabisch and P. Miranda. Exact bounds of the Möbius inverse of monotone set functions. working paper, 2013.

23. P. Miranda and M. Grabisch. Optimization issues for fuzzy measures. Int. J. of Uncertainty, Fuzziness, and Knowledge-Based Systems, 7(6):545-560, 1999.

24. P. Miranda, M. Grabisch, and P. Gil. p-symmetric fuzzy measures. Int. J. of Uncertainty, Fuzziness, and Knowledge-Based Systems, 10 (Suppl.):105-123, 2002.

25. P. Miranda and M. Grabisch. p-symmetric bi-capacities. Kybernetika, 40(4):421-440, 2004.

26. U. Faigle and W. Kern. The Shapley value for cooperative games under precedence constraints. Int. J. of Game Theory, 21:249-266, 1992.

27. E. Algaba, J. M. Bilbao, P. Borm, and J. J. López. The position value for union stable systems. Math. Meth. Oper. Res., 52:221-236, 2000.

28. U. Faigle and M. Grabisch. A discrete Choquet integral for ordered systems. Fuzzy Sets and Systems, 168:3-17, 2011. DOI 10.1016/j.fss.2010.10.003.

29. U. Faigle, M. Grabisch, and M. Heyne. Monge extensions of cooperation and communication structures. European Journal of Operational Research, 206:104-110, 2010. 10.1016/j.ejor.2010.01.043.

30. A. Honda and M. Grabisch. An axiomatization of entropy of capacities on set systems. Eur. J. of Operational Research, 190:526-538, 2008.

31. F. Lange and M. Grabisch. Values on regular games under Kirchhoff's laws. Mathematical Social Sciences, 58:322-340, 2009. DOI: 10.1016/j.mathsocsci.2009.07.003.

32. J.-P. Barthélemy. Monotone functions on finite lattices: an ordinal approach to capacities, belief and necessity functions. In J. Fodor, B. De Baets, and P. Perny, editors, Preferences and Decisions under Incomplete Knowledge, pages 195-208. Physica Verlag, 2000.

33. C. Zhou. Belief functions on distributive lattices. Artificial Intelligence, 201:1-31, 2013.

34. J. Šipoš. Integral with respect to a pre-measure. Math. Slovaca, 29:141-155, 1979.

35. M. Grabisch. The Möbius function on symmetric ordered structures and its application to capacities on finite sets. Discrete Mathematics, 287(1-3):17-34, 2004.

36. M. Grabisch. The symmetric Sugeno integral. Fuzzy Sets and Systems, 139:473-490, 2003.

37. I. Singer. Extensions of functions of $0-1$ variables and applications to combinatorial optimization. Numerical Functional Analysis and Optimization, 7(1):23-62, 1984.

38. J.-L. Marichal. An axiomatic approach of the discrete Choquet integral as a tool to aggregate interacting criteria. IEEE Tr. on Fuzzy Systems, 8(6):800-807, 2000.

39. J.-L. Marichal, P. Mathonet, and E. Tousset. Mesures floues définies sur une échelle ordinale. working paper, 1996.

40. R. Mesiar. k-order pan-additive discrete fuzzy measures. In 7th IFSA World Congress, pages 488-490, Prague, Czech Republic, June 1997.

41. P. Benvenuti, R. Mesiar, and D. Vivona. Monotone set functions-based integrals. In E. Pap, editor, Handbook of Measure Theory, pages 1329-1379. Elsevier Science, 2002.

42. M. Couceiro and J.-L. Marichal. Axiomatizations of Lovász extensions of pseudo-boolean functions. Fuzzy Sets and Systems, 181:28-38, 2011.

43. D. Schmeidler. Integral representation without additivity. Proc. of the Amer. Math. Soc., 97(2):255-261, 1986.

44. L. de Campos and M. J. Bolaños. Characterization and comparison of Sugeno and Choquet integrals. Fuzzy Sets \& Systems, 52:61-67, 1992.

45. T. Murofushi, M. Sugeno, and M. Machida. Non-monotonic fuzzy measures and the Choquet integral. Fuzzy Sets and Systems, 64:73-86, 1994.

46. M. Couceiro and J.-L. Marichal. Axiomatizations of quasi-Lovász extensions of pseudo-boolean functions. Aequat. Math., 82:213-231, 2011.

47. M. Couceiro and J.-L. Marichal. Discrete integrals based on comonotonic modularity. Axioms, 3:390-403, 2013. 
48. J.-L. Marichal. On Sugeno integral as an aggregation function. Fuzzy Sets and Systems, 114:347-365, 2000.

49. M. Sugeno. A note on derivatives of functions with respect to fuzzy measures. Fuzzy Sets and Systems, 222:1-17, 2013.

50. M. Sugeno. A way to choquet calculus. IEEE Tr. on Fuzzy Systems, to appear.

51. S. Weber. $\perp$-decomposable measures and integrals for archimedean t-conorms $\perp$. J. Math. Anal. Appl., 101:114-138, 1984.

52. R. Kruse. Fuzzy integrals and conditional fuzzy measures. Fuzzy Sets ES Systems, 10:309-313, 1983.

53. M. Sugeno and T. Murofushi. Pseudo-additive measures and integrals. J. Math. Anal. Appl., 122:197-222, 1987.

54. T. Murofushi and M. Sugeno. Fuzzy t-conorm integrals with respect to fuzzy measures : generalization of Sugeno integral and Choquet integral. Fuzzy Sets 83 Systems, 42:57-71, 1991.

55. E. P. Klement, R. Mesiar, and E. Pap. Triangular Norms. Kluwer Academic Publishers, Dordrecht, 2000.

56. W. Sander and J. Siedekum. Multiplication, distributivity and fuzzy integral I. Kybernetika, 41:397-422, 2005.

57. W. Sander and J. Siedekum. Multiplication, distributivity and fuzzy integral II. Kybernetika, 41:469-496, 2005.

58. W. Sander and J. Siedekum. Multiplication, distributivity and fuzzy integral III. Kybernetika, 41:497-518, 2005.

59. N. Shilkret. Maxitive measure and integration. Nederl. Akad. Wetensch. Proc. Ser. A, 74:109-116, 1971.

60. E. P. Klement, R. Mesiar, and E. Pap. A universal integral as common frame for choquet and sugeno integral. IEEE Tr. on Fuzzy Systems, 18:178-187, 2010.

61. E. P. Klement, R. Mesiar, F. Spizzichino, and A. Stupňanová. Universal integrals based on copulas. Fuzzy Optimization and Decision Making, 13:273-286, 2014.

62. Y. Azrieli and E. Lehrer. Extendable cooperative games. J. Public Economic Theory, 9:1069-1078, 2007.

63. E. Lehrer and R. Teper. The concave integral over large spaces. Fuzzy Sets and Systems, 159:2130-2144, 2008.

64. E. Lehrer. A new integral for capacities. Economic Theory, 39:157-176, 2009.

65. Y. Even and E. Lehrer. Decomposition-integral: Unifying choquet and the concave integrals. Economic Theory, 56:33-58, 2014. 